Working Paper

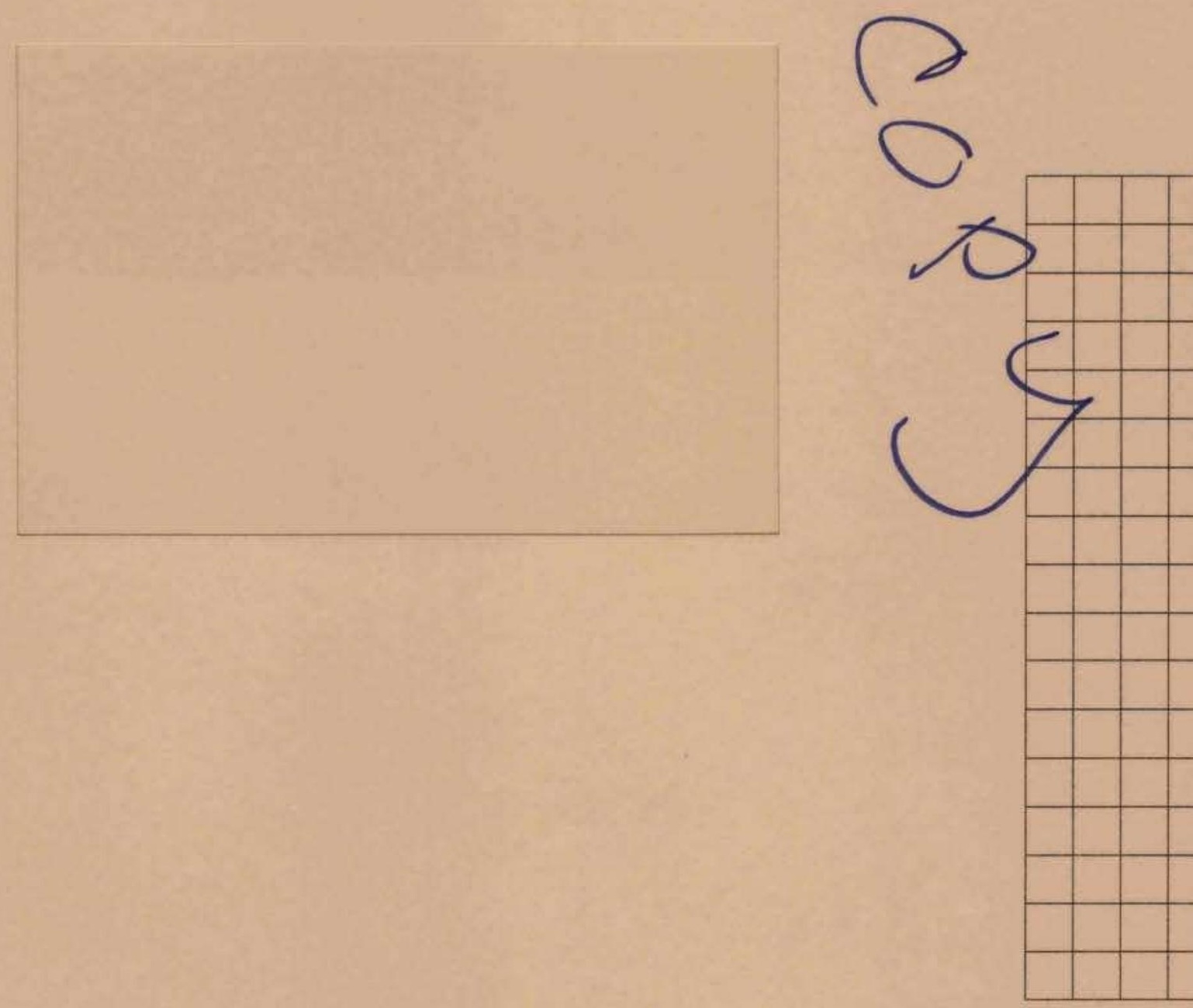

Research Department Federal Reserve Bank of Minneapolis 
Federal Reserve Bank of Minneapolis

Research Department

\section{DETERMINACY OF EQUILIBRIA IN DYNAMIC MODELS WITH \\ FINITELY MANY CONSUMERS}

Timothy J. Kehoe, David K. Levine, and Paul M. Romer*

Working Paper 400

Revised October 1988

NOT FOR DISTRIBUTION

WITHOUT AUTHOR APPROVAL

\section{ABSTRACT}

We consider a production economy with a finite number of heterogeneous, infinitely lived consumers. We show that, if the economy is smooth enough, equilibria are locally unique for almost all endowments. We do so by converting the infinite dimensional fixed point problem stated in terms of prices and commodities into a finite dimensional Negishi problem involving individual weights in a social value function. By adding a set of artificial fixed factors to utility and production functions, we can write the equilibrium conditions equating spending and income for each consumer entirely in terms of time zero factor endowments and derivatives of the social value function.

*Kehoe, Federal Reserve Bank of Minneapolis, and Department of Economics, University of Minnesota; Levine, Department of Economics, University of California, Los Angeles; Romer, Department of Economics, University of Chicago. We are grateful for conversations with Manuel Santos, Lionel McKenzie, and Andreu Mas-Colell. N.S.F. grants SES 85-09484, SES 83-20007, and SES 86-18325 and the U.C.L.A. Academic Senate provided financial support.

The views expressed herein are those of the author and not necessarily those of the Federal Reserve Bank of Minneapolis or the Federal Reserve System. This paper is preliminary and is circulated to stimulate discussion. It is not to be quoted without the author's permission. 


\section{Introduction}

It is now well established that economic models with a finite number of goods have equilibria that are generically determinate; a specification of preferences, endowments, and technological opportunities almost always (in a sense that can be made precise) determines a finite set of equilibrium outcomes that can be observed. In models with an infinite number of goods, however, equilibria can be indeterminate. For example, Kehoe and Levine (1987) provide a simple example of an overlapping generations model (without fiat money) that has a continuum of equilibria. This continuum is robust in the sense that it persists even when the basic parameters of preferences and the technology are altered, and it arises in a model that is otherwise well behaved. It has no cycles and no chaos; in fact, all of the equilibria converge asymptotically to the same stable stationary state. All of the equilibria are pareto efficient; the value of the aggregate endowment is finite; and the price sequences lie in the dual space of the commodity space. More strongly, Kehoe, Levine, Mas-Colell, and Zame (1986) show that robust indeterminacy can arise when the price and commodity spaces are the same Hilbert space.

Models with indeterminacy are undesirable from a scientific point of

view. Starting from the fundamentals of the economy, a theory based on this kind of model offers little guidance about should be observed. Nor is it possible to condition on the equilibrium values that are observed and do a comparative statics analysis; small changes in the underlying parameters can lead to large and discontinuous changes in the observed outcomes. Indeterminacy is especially troublesome in dynamic models because it undermines the interpretation of these models in terms of an equilibrium where agents trade in spot markets and form expectations about the future. If there is indeter- 
minacy concerning the equilibrium that obtain in the present, there also is indeterminacy in the equilibria starting from future dates, and this makes expectation formation problematic.

In this paper, we extend the class of dynamic models that are known to have a finite number of locally unique equilibria and clarify the sense in which finiteness is important for this result. As noted above, models with a finite number of goods are generically determinate, but this is of no help for the study of infinite horizon dynamic models. Moreover, we know from examples that a finite number of goods is not necessary for determinacy. A model with a single representative agent is determinate regardless of the number of goods in the model; the equilibrium quantities are solutions to a concave maximization problem, and under mild strict concavity assumptions they, are unique. The Kehoe-Levine overlapping generations example shows that indeterminacy can arise in a model with both an infinite number of goods and an infinite number of individuals. What the results presented here suggest is that this kind of double infinity of goods and consumers is the crucial element in this example. In particular, we show that what matters for determinacy in the usual representative agent dynamic model is not that there be a single agent in the economy. It is sufficient for the number of agents to be finite.

Proofs of determinacy in a model with a finite number of goods proceed by examining the properties of a finite number of supply-equals-demand equations that depend on a finite number of prices. Determinacy follows from essentially counting equations and unknowns. Since the number of equations is the same as the number of undetermined prices, there are almost always a finite number of solutions to these equations. Counting equations and unknowns does not work when there are an infinite number of equations and variables. What we exploit is a duality relationship between goods and individ- 
uals. We reduce the specification of the equilibrium to a finite number of equations, one for each individual, that equate the value of consumption with the value of the individual's endowment. These equations depend on a set of welfare weights that are the analogs of prices; the weights are assigned to individuals in a pareto optimization problem that determines consumption for each individual by maximizing the weighted sum of individual utilities. Our analysis of determinacy then proceeds as in the usual finite dimensional case.

Our results extend those of Muller and Woodford (1986), who consider production economies with both finitely and infinitely lived consumers. They show that there can be no indeterminacy if the infinitely lived consumers are sufficiently large. Their results are local, however, and concern only equilibrium that converge to a particular stationary state. We prove a global theorem: for a given starting capital stock, there are only finitely many equilibria. (We do not, however, permit an infinite number of finitely lived consumers, as do Muller and Woodford.)

We assume that markets are complete and that the technology and preferences are convex. Consequently, the behavior of equilibria in our model can be characterized by the properties of a value function. This is because the second theorem of welfare economics holds: any pareto efficient allocation can be decentralized as a competitive equilibrium with transfer payments. If the preferences of consumers can be represented by concave utility functions, then an equilibrium with transfers can be calculated by maximizing a weighted sum of the individual utility functions subject to the feasibility constraints implied by the aggregate technology and the initial endowments. Showing that an equilibrium exists is equivalent to showing that there exists a vector of welfare weights such that the transfer payments needed to decentralize the resulting pareto efficient allocation are zero. This approach has 
been pioneered by Negishi (1960) and applied to dynamic models by Bewley (1980,1982). Using this approach, Kehoe and Levine (1985a) have considered the regularity properties of an infinite horizon economy without production.

In general, calculating the transfers associated with a given set of weights requires the complete calculation of equilibrium quantities and prices. In a dynamic model with an infinite number of commodities, this can be awkward. To simplify the calculation, we adopt an alternative strategy based on the simple geometrical observation that any convex set in $\mathbf{R}^{\mathrm{n}}$ can be interpreted as the cross section of a cone in $R^{n+1}$. To exploit this fact, we add a set of artificial fixed factors to the economy and include them as arguments of the weighted social value function. These factors are chosen so that the augmented utility and production functions are homogeneous of degree one. Thus, the usual problem of choosing a point on the frontier of a convex utility possibility set is converted into a problem of choosing a point from a cone of feasible values for utility. This extension has theoretical advantages analogous to those that arise when a strictly concave production function is converted into a homogeneous of degree one function by the addition of a fixed factor. When the technology for the firm is a cone, profits and revenues are completely accounted for by factor payments. Analogously, making the social value function homogeneous of degree one simplifies the accounting necessary to keep track of the transfers associated with any given pareto efficient allocation. The present value of income and expenditure for each individual can be calculated directly from an augmented list of endowments and from the derivatives of the augmented social value function without explicitly calculating the dynamic paths for prices or quantities. This is the framework for studying multiagent intertemporal equilibria developed by Kehoe and Levine $(1985 b)$. 
In such a setting, equilibria are equivalent to zeros of a simple finite dimensional system of equations involving the derivatives of the social value function and the endowments. Intuition says that, since the number of equations and the number of unknowns in this system are both equal to the number of agents, equilibria ought to be determinate. To do the usual kind of regularity analysis, however, we require that the functions involved in the system of equations that determines the equilibria be continuously differentiable. Because these functions involve derivatives of the social value function, they are continuously differentiable if the value function is twice continuously differentiable. Thus, we show how to reduce an equilibrium problem to a problem in growth theory: how smooth is the value function? Roughly, the result is the same as in the finite case: if the economy (in this case, the value function) is smooth enough, equilibria are generically determinate. Unlike the finite horizon case, however, we have no simple and general assumptions on preferences and technology that guarantee that the economy is smooth enough.

In our basic model, a one sector neoclassical growth model, smoothness of the value function may be deduced from a global turnpike theorem. With multiple capital stocks, twice continuous differentiability of the value function is not known to follow from smoothness of utility and production possibilities, except in special cases. If the discount factor is sufficiently close to one, then Araujo and Scheinkman (1977) show that the value function must be twice continuously differentiable; if the discount factor is sufficiently close to zero, Boldrin and Montrucchio (1987) show that the value function must be twice continuously differentiable. In the latter case, a global turnpike theorem is not true, and Boldrin and Montrucchio (1986) and Deneckere and Pelikan (1985) have shown that both cycles and chaos can occur 
with small discount factors. In fact, Boldrin and Montrucchio describe a general method for constructing examples with $C^{\infty}$ smoothness and chaos. Consequently, determinacy does not rest on a turnpike theorem, but on the rather different assumption of a twice continuously differentiable value function.

In the case of intermediate values of the discount factor relatively little is known. Boldrin and Montrucchio (19.88) provide some conditions sufficient for a $\mathrm{C}^{2}$ value function. In addition, Kehoe, Levine, and Romer (1987) show that regardless of the discount factor, there are at most finitely many equilibria that converge to a nondegenerate steady state. Moreover, our general methods apply to stochastic as well as deterministic complete contingent claims economies (see Kehoe and Levine 1985b), and results due to Blume, Easley, and O'Hara (1982) imply that a small amount of the right kind of uncertainty leads to smooth value functions. This can provide an alternative direction for proving determinacy results.

In the next section, we set up a simple one sector, multiperson economy. Section 3 characterizes equilibria as social optima without transfer payments. Section 4 analyzes properties of the savings function of the economy. Section 5 shows how differentiability of the savings function implies determinacy of equilibria. Finally, Section 6 discusses extensions to multisector models.

\section{The Basic Model}

Consider a simple m person neoclassical growth model. The preferences of each consumer take the usual additively separable form, discounted by the common factor $\beta, 0<\beta<1$. Consumption of the single perishable good by consumer $i$ at time $t$ is denoted by $c_{i t}$. The utility function for consumer $i$, $i=1, \ldots, m$, is then $\sum_{t=0}^{\infty} \beta^{t} u_{i}\left(c_{i t}\right)$. The initial endowment of capital, the single reproducible productive factor, is $\bar{k}_{0}>0$, and $\theta_{i}>0$ is the share 
owned by consumer i. Obviously, $\sum_{i=1}^{m}{ }_{i}=1$, and $\theta_{i} \bar{k}_{0}$ is the endowment of consumer i. The economy also has $\bar{x}>0$ units of labor in each period, a perishable productive factor; $\phi_{i}>0$ is the share of total labor owned by consumer i. Again, $\sum_{i=1}^{m} \phi_{i}=1$, and $\phi_{i} \bar{x}$ is the endowment of consumer $i$. If $q_{t}$ denotes the output of consumption, and $k_{t}$ and $x_{t}$ inputs of capital and labor, the technology is described by an aggregate production function $q_{t}+k_{t+1}=$ $G\left(k_{t}, x_{t}\right)$.

We next specify the properties assumed for the preferences and technology. The assumptions concerning continuity, monotonicity, and concavity are standard.

Assumption 1: For 1, $\ldots, \mathrm{m}, \mathrm{u}_{\mathrm{i}}: \mathbf{R}_{+} \rightarrow \mathbf{R} \cup\{-\infty\}$ is concave, strictly increasing, and continuous. On the strictly positive orthant, $\mathbf{R}_{++}, u_{i}$ is smooth (infinitely many times continuously differentiable) and $\partial u_{i} / \partial c>0$, $\partial^{2} u_{i} / \partial^{2} c<0$. Moreover, $\lim _{c \rightarrow 0} \partial u_{i}(c) / \partial c=+\infty$.

In the statement that $u_{i}$ is continuous, we are using the natural topology on $R \cup\{-\infty\}$, that is, the one generated by adding open intervals of the form $[-\infty, a)$ to the usual topology on $\mathbf{R}$. For example, the functions $u(c)=$ In $c$ and $u(c)=\left(c^{0}-1\right) / p, \rho \leq 1$ all satisfy the conditions of this assumption.

Assumption 2: The production function $G: R_{+}^{2}+R_{+}$is homogeneous of degree one, concave, and continuous. On the strictly positive orthant, $\mathrm{R}_{++}^{2}, \mathrm{G}$ is smooth, and $\partial G / \partial k>0, \partial G / \partial x>0, \partial^{2} G / \partial k^{2}<0 . \quad$ Moreover, $G(0, x)=0$, $\lim \partial G(k, \bar{x}) / \partial k=+\infty$, and $\lim \sup G(k, \bar{x}) / k<1$. $k \rightarrow 0$ $k \rightarrow \infty$

In what follows, smoothness plays an important role. To ensure that it holds, we need know not only that the relevant functions be smooth, but also that production and consumption plans are strictly interior. This fol- 
lows from the infinite steepness conditions, $\partial u_{i}(0) / \partial c=+\infty$, and $\partial G(0, \bar{x}) / \partial k=\infty$. Finally, by assuming $\lim \sup G(k, \bar{x}) / k<1$, we ensure that the capital stock must remain bounded.

\section{Characterization of Equilibrium}

A competitive equilibrium for this model consists of a sequence $p_{0}$, $\mathrm{p}_{1}, \ldots$, of prices for the consumption good, a price $r$ for the initial capital stock, a sequence $w_{0}, w_{1}, \ldots$ of prices for labor, a consumption allocation $c_{i 0}, c_{i 1}, \ldots$ for each consumer $i$, a sequence of capital stocks $k_{0}, k_{1}, \ldots$, a sequence of labor inputs $x_{0}, x_{1}, \ldots$, and a sequence of outputs of the consumption good $q_{0}, q_{1}, \ldots$. Given the prices $p_{t}, w_{t}$, and $r$, the consumption allocation $c_{i t}$ must solve the utility maximization problem for consumer $i$ :

$$
\max \sum_{t=0}^{\infty} \beta^{t} u_{i}\left(c_{i t}\right)
$$

subject to

$$
\sum_{t=0}^{\infty} p_{t} c_{i t} \leq \theta_{i} r \bar{k}_{0}+\phi_{i} \sum_{t=0}^{\infty} w_{t} \bar{x} .
$$

Furthermore, given the prices $p_{t}$, the production plans $k_{t}, x_{t}, q_{t}$ must maximize profits:

$$
\max \sum_{t=0}^{\infty}\left(p_{t} q_{t}-w_{t} x_{t}\right)-r k_{0}
$$

subject to

$$
q_{t}+k_{t+1} \leq G\left(k_{t}, x_{t}\right), t=0,1, \ldots .
$$

Finally, demand must equal supply for the consumption good and labor in every period and for the initial capital stock:

$$
\sum_{i=1}^{m} c_{i t}=q_{t}, t=0,1, \ldots
$$




$$
\begin{aligned}
& x_{t}=\bar{x} \\
& k_{0}=\bar{k}_{0} .
\end{aligned}
$$

As is usual in such capital theory problems, the construction of a competitive equilibrium is accomplished by solving a social optimization problem. The necessary conditions for this problem guarantee the existence of a set of shadow prices that satisfy certain properties. It is then a trivial matter to show that these prices, along with the optimal quantities, satisfy the sufficient conditions for the optimization problems of the consumer and the firm.

Consider the social planning problem of determining a pareto efficient consumption allocation and production sequence. Given nonnegative welfare weights $\left(\alpha_{1}, \alpha_{2}, \ldots, \alpha_{m}\right)$, we maximize a weighted sum of the individual consumers' utilities subject to feasibility constraints:

$$
\max \sum_{i=1}^{m} \alpha_{i} \sum_{t=0}^{\infty} \beta^{t} u_{i}\left(c_{i t}\right)
$$

subject to

$$
\begin{aligned}
& \sum_{i=1}^{m} c_{i t}+k_{t+1} \leq G\left(k_{t}, x_{t}\right), t=0,1, \ldots \\
& x_{t} \leq \bar{x}, t=0,1, \ldots \\
& k_{0} \leq \bar{k}_{0} \\
& k_{t} \geq 0, t=0,1, \ldots .
\end{aligned}
$$

Using results that are analogous with the Kuhn-Tucker Theorem for finite maximization problems, we can express the necessary conditions for this problem as a set of intertemporal optimization conditions and a transversality condition at infinity. Let $p_{t}$ be the Lagrange multiplier applied to the 
constraint on output in each period, let $w_{t}$ be the multiplier on the constraint on $x_{t}$, and let $r$ be the multiplier on the initial stock of capital. The Lagrangean for this problem is

$$
\begin{aligned}
f= & \sum_{i=1}^{m} \alpha_{i} \sum_{t=0}^{\infty} \beta^{t} u_{i}\left(c_{i t}\right)+r\left(\bar{k}_{0}-k_{0}\right) \\
& +\sum_{t=0}^{\infty}\left[w_{t}\left(\bar{x}-x_{t}\right)+p_{t}\left(G\left(k_{t}, x_{t}\right)-k_{t+1}-\sum_{i=1}^{m} c_{i t}\right)\right] .
\end{aligned}
$$

Since the optimal qualities $c_{i t}, k_{t}, x_{t}$ are strictly positive, the intertemporal optimization conditions follow by setting derivatives of $f$ equal to zero:

$$
\begin{aligned}
& \alpha_{i} \beta^{t} \partial u_{i}\left(c_{i t}\right) / \partial c-p_{t}=0, i=1, \ldots, m ; t=0,1, \ldots \\
& p_{0} \partial G\left(k_{0}, x_{0}\right) / \partial k-r=0, \\
& -p_{t-1}+p_{t} \partial G\left(k_{t}, x_{t}\right) / \partial k=0, t=1,2, \ldots \\
& p_{t} \partial G\left(k_{t}, x_{t}\right) / \partial x-w_{t}=0, t=0,1, \ldots \\
& x_{t}=\bar{x} \\
& k_{0}=\bar{k}_{0} .
\end{aligned}
$$

The transversality condition at infinity is

$$
\lim _{t \rightarrow \infty} p_{t} k_{t+1}=0
$$

See Weitzman (1973) or Romer and Shinotsuka (1988).

The sufficient conditions for the problem of the consumer and the problem of the firm can be derived analogously. Let $\lambda_{i}$ denote the multiplier on the budget constraint for consumer $i$, when faced with prices $p_{t}$. The sufficient conditions for an optimal consumption sequence are

$$
\beta^{t} \partial u_{i}\left(c_{i t}\right) / \partial c-\lambda_{i} p_{t}=0, t=0,1, \ldots
$$


combined with the requirement of overall budget balance,

$$
\sum_{t=0}^{\infty} p_{t} c_{i t}=\theta_{i} r \bar{k}_{0}+\phi_{i} \sum_{t=0}^{\infty} w_{t} \bar{x} .
$$

Notice that the left hand side of this expression, expenditure on consumption, is strictly decreasing in $\lambda_{i}$ and that the right hand side is given, so that this expression can be thought of as determining $\lambda_{i}$.

Let $\mu_{t}$ denote the Lagrange multiplier associated with the constraint faced by the firm. Sufficient conditions for the firm's maximization problem are once again a set of intertemporal conditions and a transversality condition at infinity. Using once again the fact that the optimal quantity choices are interior, we can derive the intertemporal conditions by setting derivatives of this expression equal to zero:

$$
\begin{aligned}
& p_{t}-\mu_{t}=0, t=0,1, \ldots \\
& -r+\mu_{0} \partial G\left(k_{0}, x_{0}\right) / \partial k=0 \\
& -\mu_{t-1}+\mu_{t} \partial G\left(k_{t}, x_{t}\right) / \partial k=0, t=1,2, \ldots \\
& -w_{t}+\mu_{t} \partial G\left(k_{t}, x_{t}\right) / \partial x=0, t=0,1, \ldots .
\end{aligned}
$$

The transversality condition is

$$
\lim _{t \rightarrow \infty} \mu_{t} k_{t+1}=0 \text {. }
$$

These conditions can be simplified to

$$
\begin{aligned}
& \partial G\left(k_{0}, \bar{x}\right) / \partial k_{0}=\frac{r}{p_{0}} \\
& \partial G\left(k_{t}, \bar{x}\right) / \partial k_{t}=\frac{p_{t-1}}{p_{t}}, t=1,2, \ldots
\end{aligned}
$$




$$
\begin{aligned}
& \partial G\left(k_{0}, \bar{x}\right) / \partial x=\frac{w_{t}}{p_{t}}, t=0,1, \ldots \\
& \lim _{t \rightarrow \infty} p_{t} k_{t+1}=0 .
\end{aligned}
$$

Comparing the necessary conditions for the weighted social optimization problem with the sufficient conditions for the consumer and firm problems, we observe that the quantities from a competitive equilibrium are pareto efficient. They solve the social optimization problem when the weights $\alpha_{i}$ are chosen to satisfy $\alpha_{i}=1 / \lambda_{i}$. This is simply the first welfare theorem for this economy. Notice too that for any arbitrary weights $\alpha_{i}$, the quantities from the social optimization problem can be decentralized as a competitive equilibrium with transfers. All that is required is to adjust the multiplier $\lambda_{i}$ representing the marginal utility of income for each individual so that it equals that individual's weight $\alpha_{i}$; as we have remarked above, $\lambda_{i}$ for each individual varies monotonically with the income allocated to individual i. This is, of course, the second welfare theorem for this economy. The appropriate transfer to each individual is that amount that just allows the individual to afford the consumption stream allocated by the social optimization problem. Thus, for given weights $a=\left(a_{1}, a_{2}, \ldots, a_{m}\right)$, the required transfers are

$$
\sum_{t=0}^{\infty} p_{t}(\alpha) c_{i t}(\alpha)-\theta_{i} r(\alpha) \bar{k}_{0}-\phi_{i} \sum_{t=0}^{\infty} w_{t}(\alpha) \bar{x}, i=1, \ldots, m .
$$

For this economy, a competitive equilibrium in the usual sense corresponds to a set of weights $\alpha$ such that these transfer to zero.

In principal, this gives all we need to consider the regularity properties of this economy. By equating the transfer payments for each of the $m$ consumers to 0 , we have $m$ equations in the $m$ unknowns, $\left(\alpha_{1}, \alpha_{2}, \ldots, \alpha_{m}\right)$. In practice, this is not a useful system of equations to work with because the 
equations require the calculation of the infinite set of quantities $c_{i t}(\alpha)$ and an infinite list of price, $p_{t}(\alpha), w_{t}(\alpha)$ for each choice of the vector $\alpha$. One could attempt to explicitly characterize the dependence of these infinite dimensional vectors on $\alpha$; in the next section, however, we show how this step can be avoided by the use of a suitably chosen value function. Of course the infinite dimensional nature of the problem does not disappear. Rather, it is embodied in the properties of the value function, which is the result of solving the social planning problem, an easier infinite dimensional problem than the original equilibrium problem.

\section{The Savings Function}

Let us now develop a characterization of solutions to the social planning problem, and of competitive equilibria, in dynamic programming terms. Given an aggregate endowment of capital $k_{0}$, a labor supply $x$, and a vector of nonnegative welfare weights $\alpha$, we define a value function $V\left(\mathrm{k}_{0}, \mathrm{x}, \alpha\right)$ as the maximum of

$$
\sum_{i=1}^{m} \alpha_{i} \sum_{t=0}^{\infty} \beta^{t} u_{i}\left(c_{i t}\right)
$$

subject to the constraints

$$
\sum_{i=1}^{m} c_{i t}+k_{t+1} \leq G\left(k_{t}, x\right), t=1,2, \ldots .
$$

The envelope theorem allows us to treat the derivative $\partial \mathrm{V}\left(\mathrm{k}_{0}, \mathrm{x}, \alpha\right) / \partial \mathrm{k}_{0}$ as the price for capital $r$ and use it to calculate the value of the capital endowment $\theta_{i} \overline{\mathrm{k}}_{0}$ for each individual. Similarly, we can show that

$$
\partial V\left(k_{0}, \bar{x}, \alpha\right) / \partial x=\sum_{t=0}^{\infty} w_{t},
$$


so we can calculate the present value of the labor endowment $\phi_{i} \bar{x}$ for each individual. To calculate the transfers associated with these weights, we must also calculate the expenditure of each individual.

To calculate individual expenditures, we must introduce an accounting device. We first show that strictly concave utility functions can be made homogeneous of degree one. In production theory a decreasing returns technology can be converted into a constant returns technology by introducing a fixed factor to act as an accounting device to keep track of producer surplus--the difference between revenue and expenditure (see, for example, McKenzie 1959). A similar factor can be used to account for consumer surplus --the difference between utility and expenditure. Introduce an additional, person specific fixed utility factor $y_{i}$ for each agent, and endow agent $i$ with the entire aggregate supply of one unit of factor i. (For simplicity, we make no distinction in the notation between the individual's holdings of factor $y_{i}$ and the aggregate endowment.) As in production theory, for $y_{i}>0$, define an augmented utility function $U_{i}\left(c, y_{i}\right)=y_{i} u_{i}\left(c / y_{i}\right)$. We now define a value function $\mathrm{V}\left(\mathrm{k}_{0}, \mathrm{x}, \mathrm{y}, \alpha\right)$ as the maximum of the weighted sum of the augmented utility functions subject to the augmented technology.

If we let $c_{i t}$ denote the optimal consumption of agent $i$ at time $t$, the first order conditions from the maximization imply the equality

$$
\beta^{t} \alpha_{i} \partial U_{i}\left(c_{i t}, y_{i}\right) / \partial c=\beta^{t} \alpha_{j} \partial U_{j}\left(c_{j t}, y_{j}\right) / \partial c
$$

As a result, weighted discounted marginal utility for any consumer can be used as a present value price for consumption at time $t$. The only difference from the usual representative consumer framework is that the weights a convert the individual marginal utility prices into a social marginal value price. We can then evaluate the expenditure of consumer $i$ in period $t$ as $c_{i t}$ multiplied by 
this price. Using the properties of homogeneous functions, we can decompose period $t$ utility for consumer $i$ into the sum of a term of this form and an analogous term involving the added utility factor:

$$
U_{i}\left(c_{i t}, y_{i}\right)=c_{i t} \partial U_{i}\left(c_{i t}, y_{i}\right) / \partial c+y_{i} \partial U_{i}\left(c_{i t}, y_{i t}\right) / \partial y .
$$

If the term involving the utility factor is interpreted as a measure of consumer surplus, expenditure on goods in period t is simply utility minus consumer surplus. Using the envelope theorem, we can then calculate the present value of consumer surplus for agent $i$ as the derivative of the social value function $\mathrm{v}\left(\mathrm{k}_{0}, \mathrm{x}, \mathrm{y}, \alpha\right)$ with respect to $\mathrm{y}_{\mathrm{i}}$ multiplied by the endowment $\mathrm{y}_{\mathrm{i}}$ :

$$
y_{i} \partial V\left(k_{0}, x, y, \alpha\right) / \partial y_{i}=\sum_{t=0}^{\infty} \beta^{t} \alpha_{i} y_{i} \partial U_{i}\left(c_{i t}, y_{i}\right) / \partial y_{i} .
$$

Similarly, we can calculate the discounted sum of utility for consumer i, measured in social value units, as the derivative (we show below that $V$ is differentiable) of the social value function with respect to $\alpha_{i}$ multiplied by $\alpha_{i}:$

$$
\alpha_{i} \partial V\left(k_{0}, x, y, \alpha\right) / \partial \alpha_{i}=\sum_{t=0}^{\infty} \beta^{t} \alpha_{i} U_{i}\left(c_{i t}, y_{i}\right) .
$$

Then the present value of expenditure by agent $i$ is simply the difference

$$
\alpha_{i} \partial V\left(k_{0}, x, y, \alpha\right) / \partial \alpha_{i}-y_{i} \partial V\left(k_{0}, x, y, \alpha\right) / \partial y_{i},
$$

The transfer to agent i necessary to support this equilibrium is zero if and only if this expenditure is equal to the time zero value of the agent's endowment,

$$
\theta_{i} k_{0} \partial V\left(k_{0}, x, y, \alpha\right) / \partial k_{0}+\phi_{i} \bar{x} \partial V\left(k_{0}, x, y, \alpha\right) / \partial x .
$$


Formally, equality of these two expressions can be interpreted in terms of an augmented economy where trade in the utility factors $y_{i}$ actually takes place. In this case, this equality can be interpreted as a requirement that the value of the augmented endowment for agent $i, \theta_{i} \bar{k}_{0} \partial V / \partial k_{0}+\phi_{i} \bar{x} \partial V / \partial x+$ $y_{i} \partial V / \partial y_{i}$, equals the amount of social utility purchased, $\alpha_{i} \partial V / \partial \alpha_{i}=$ $\alpha_{i} \sum_{t=0}^{\infty}{ }^{t^{t} U_{i}}$.

It is useful to define a net savings function $s_{i}$ for consumer $i$ as

$$
\begin{aligned}
s_{i}\left(k_{0}, \theta, \phi, \alpha\right)= & \theta_{i} k_{0} \partial V\left(k_{0}, \bar{x}, 1, \alpha\right) / \partial k_{0}+\phi_{i} \bar{x} \partial V\left(k_{0}, \bar{x}, 1, \alpha\right) / \partial x \\
& +y_{i} \partial V\left(k_{0}, \bar{x}, 1, \alpha\right) / \partial y_{i}-\alpha_{i} \partial V\left(k_{0}, \bar{x}, 1, \alpha\right) / \partial \alpha_{i} .
\end{aligned}
$$

For a given set of welfare weights $\alpha$, the transfer for each individual needed to support the social optimum as a competitive equilibrium is the negative of the net savings for that individual. A competitive equilibrium is therefore equivalent to a vector of weights a such that the vector $s\left(\bar{k}_{0}, \theta, \phi, \alpha\right)=0$.

To calculate equilibria, we need to analyze the savings function. our goal is to prove the following results:

Proposition 1: Under Assumptions 1 and 2:

(a) $s\left(k_{0}, \theta, \phi, \alpha\right)$ is homogeneous of degree one in $\alpha$.

(b) $\sum_{i=1}^{m} s_{i}\left(k_{0}, \theta, \phi, \alpha\right)=0$.

(c) For each $\mathrm{k}_{0}, \theta, \phi, \lim _{\alpha_{i} \rightarrow 0} s_{i}\left(k_{0}, \theta, \phi, \alpha\right)>0$.

(d) $s$ is continuously differentiable. It is affine in both $\theta$ and $\phi$ and $D_{\theta} S$ is diagonal and nonsingular.

An implication of this proposition is that the functions $s_{i}(\alpha) / \alpha_{i}$ satisfy the same formal properties as the excess demand functions of a static pure exchange economy with $\mathrm{m}$ goods. This observation leads immediately to the conclusion that an equilibrium exists. 
In proving Proposition 1, notice that in part (d), the dependence of $s$ on $\theta$ is obvious from the definition of the savings function $\left(^{*}\right): s$ is affine in $\theta$ and $D_{\theta} S$ is a diagonal matrix with diagonal entries $\mathrm{k}_{0} \partial \mathrm{V}\left(\mathrm{k}_{0}, \overline{\mathrm{x}}, 1, \alpha\right) / \partial \mathrm{k}_{0}$. Moreover, since utility and production are strictly monotone and endowments strictly positive, these entries are strictly positive. Similarly, $s$ is affine in $\phi$.

The proof of the remaining parts of the proposition follows by using dynamic programming to characterize the value function. To solve the social optimization problem, we first solve the problem in period $t$ for given $k_{t}$ and $k_{t+1}$. Let the vector of weights $\alpha$ be fixed. Defined $w(C, y, \alpha)$ as the maximum of

$$
\max \sum_{i=1}^{m} \alpha_{i} U_{i}\left(c_{i}, y_{i}\right)
$$

subject to

$$
\sum_{i=1}^{m} c_{i}=c .
$$

The following result follows immediately from Assumption 1:

Lemma 2: The function $\mathrm{w}$ is concave in $(\mathrm{C}, \mathrm{y})$ convex in $\alpha$, strictly increasing in $C$ and $\alpha$, and continuous. On the strictly positive orthant $w$ is smooth and $\partial w / \partial C>0, \partial^{2} w / \partial C^{2}<0$. Moreover, $\partial w(0, y, \alpha) / \partial C=+\infty$. The function $w$ is homogeneous of degree one in $(\mathrm{C}, \mathrm{y})$ and separately in $\alpha$.

Using the function $w$, we can write social value in period $t$ as

$$
v\left(k_{t}, k_{t+1}, x, y, \alpha\right)=w\left(G\left(k_{t}, x\right)-k_{t+1}, y, \alpha\right) .
$$

The social present value function $V$ then satisfies the dynamic programming relationship 


$$
V\left(k_{t}, x, y, a\right)=\max _{k_{t+1}} v\left(k_{t}, k_{t+1}, x, y, \alpha\right)+\beta V\left(k_{t+1}, x, y, \alpha\right) .
$$

Proof of Proposition 1: Notice that $\mathrm{v}$ is concave and homogeneous of degree one in $\left(k_{t}, k_{t+1} x, y\right)$ and convex and homogeneous of degree one in $a$. It follows directly that $V$ shares these same properties. An argument of Benveniste and Scheinkman (1979) implies that it is also continuously differentiable. Examining (*), we see that $s_{i}$ is made up of terms where a constant is multiplied by $\partial V / \partial z$, for arguments $z$ other than $\alpha$, and of $a$ term $\alpha_{i} \partial V / \partial \alpha_{i}$. In either case, each term is homogeneous of degree one, since $V$ is homogeneous of degree one in $\alpha$. This proves part (a) of the proposition. Moreover, since $\mathrm{V}$ is continuously differentiable, homogeneity of $V$ of degree one in $k_{t}$, $x$, and $y$ and $\sum_{i} \theta_{i}=1, \sum_{i} \phi_{i}=1$ imply that the terms

$$
\theta_{i} k_{0} \partial V / \partial k_{0}+\phi_{i} \bar{x} \partial V / \partial x+y_{i} \partial V / \partial y_{i}
$$

add up to $\mathrm{V}\left(\mathrm{k}_{\mathrm{t}}, \mathrm{x}, \mathrm{y}, \alpha\right)$ homogeneity of $\mathrm{V}$ of degree one in a implies that the terms $a_{i} \partial V / \partial a_{i}$ do as well. This proves part (b).

Next, let $\alpha^{\ell}$ be a sequence in the interior of the positive orthant converging to a point a such that $\alpha_{i}=0$ and $\alpha_{j} \neq 0$. If $c_{i}^{\ell}$ and $c_{j}^{l}$ denote the corresponding optimal consumption choices in the definition of $w$, infinite steepness on the boundary of the utility functions implies that $c_{i}^{\ell}$ and $c_{j}^{\ell}$ are strictly positive and that the equality

$$
a_{i}^{\ell} \partial u_{i}\left(c_{i}^{\ell}\right) / \partial c_{i}=a_{j}^{l} \partial u_{j}\left(c_{j}^{\ell}\right) / \partial c_{j}
$$

holds for all $\ell$. By an application of the maximum theorem (see, for example, Hildenbrand 1974), since the mapping that sends a to the vector of optimal consumptions is single valued, it is a continuous function. From the definition of $w$, it is clear that the optimal value for $c_{i}$ is 0 since $a_{i}=0$. By 
continuity, $c_{i}^{\ell}$ converges to zero while $a_{j}^{\ell}>0$ clearly implies $c_{j}^{\ell} \neq 0$. Using the equality noted above, this implies that

$$
\lim _{\ell \rightarrow \infty} \alpha_{i}^{\ell} c_{i}^{\ell} \partial u_{i}\left(c_{i}^{\ell}\right) / \partial c_{i}=0 .
$$

That is, the expenditure on consumption goods allowed consumer i goes to 0 as his weight in social utility goes to 0 .

By the envelope theorem, we know that the last term in the definition of $s$ is simply the product of the utility weights times the present discounted utility for each consumer. Using the homogeneity of the augmented utility function $U_{i}\left(c, y_{i}\right)$, we can combine the last two terms in $s$ and express $\mathbf{s}_{\mathrm{i}}$ as

$$
\begin{aligned}
s_{i}\left(k_{0}, \theta, \phi, \alpha\right)= & \theta_{i} k_{0} \partial V\left(k_{0}, \bar{x}, 1, \alpha\right) / \partial k_{0}+\phi_{i} \bar{x} \partial V\left(k_{0}, \bar{x}, 1, \alpha\right) / \partial x \\
& -a_{i} \sum_{t=0}^{\infty}{ }^{t} c_{i t} \partial u_{i}\left(c_{i t}\right) / \partial c_{i t} .
\end{aligned}
$$

By the argument above, the last term in this expression goes to 0 as $\alpha_{i}^{\ell}$ goes to 0 ; from the definition of $w$ and the envelope theorem, the first and second derivatives of $V$ can be expressed in terms of the marginal utility of agent $J$ and hence is continuous as $\ell \rightarrow \infty$. Therefore, $s$ approaches

$$
\theta_{i} k_{0} \partial V\left(k_{0}, \bar{x}, 1, a\right) / \partial k_{0}+\phi_{i} \partial V\left(k_{0}, \bar{x}, 1, w\right) / \partial x
$$

Notice that this last term is simply the value of consumer i's endowment of capital and labor. By assumption, $\theta_{i}, \phi_{i}>0$. Since $G$ and $u_{j}$ were assumed to be strictly increasing, every component of $\partial V / \partial k_{0}$ is strictly positive and $s_{i}$ is greater than 0 .

It remains to show that $s$ is continuously differentiable. From $\left(^{*}\right)$, it clearly suffices to show that $\mathrm{V}$ is twice continuously differentiable. In this one dimensional example, $c^{2}$ differentiability of $v$ follows from basic 
properties of the social optimization problem. Given $\theta, \phi$, and $\alpha$, the maximization problem is a standard one sector growth problem with period objective function w. By Lemma 2, this satisfies standard properties. It is well known that such a problem satisfies a global turnpike property and has no unit roots. See, for example, Harris (1987, pp. 34-45), or Cass (1965). In addition $v$ is strictly concave in $\left(k_{t}, k_{t+1}\right)$ and

$$
\frac{\partial^{2} v}{\partial k_{t} \partial k_{t+1}}\left(k_{t}, k_{t+1}, x, y, \alpha\right)=-\frac{\partial^{2} w}{\partial c^{2}}\left(G\left(k_{t}, x\right)-k_{t+1}, y, \alpha\right) \frac{\partial G}{\partial k_{t}}\left(k_{t}, x\right)>0 \text {. }
$$

Notice that $\partial^{2} v / \partial k_{t} \partial k_{t+1}$ could be negative if there was joint production. The implications of this possibility are discussed in section 6 . In a situation where it is positive and there is a global turnpike with no unit roots, Araujo and Scheinkman (1977) show that $V$ is twice continuously differentiable with respect to $k_{t}$ and $B$. A straightforward extension of their argument shows that it is also jointly twice continuously differentiable with respect to $k_{t}, x, y$, and $a$. Let $k$ denote the infinite vector $\left(k_{1}, k_{2}, \ldots\right)$ in $\ell_{\infty}$, the space of bounded sequences. The optimal path can be characterized by first order conditions $\xi\left(\mathrm{k}, \mathrm{k}_{0}, \mathrm{x}, \mathrm{y}, \alpha\right)=0$ where $\xi\left(\cdot, \mathrm{k}_{0}, \mathrm{x}, \mathrm{y}, \alpha\right): \ell_{\infty} \rightarrow_{\infty} \cdot$ Under the stated conditions Araujo and Scheinkman show that $\xi$ is continuously differentiable in $\mathrm{k}$ and $\mathrm{k}_{0}$; the Appendix of Kehoe, Levine, and Romer (1987) shows that this extends in a straightforward way to cover other parameters such as $\mathrm{x}, \mathrm{y}$, and a. Araujo and Scheinkman also show under the stated conditions that the derivative of $\xi$ with respect to $k$ is nonsingular; it follows from the implicit function theorem that the optimal $\mathrm{k}$ is a continuously differentiable function of $\mathrm{k}_{0}, \mathrm{x}, \mathrm{y}$, and $a$. Finally, observe that the first derivatives of $\mathrm{V}$ are, by the envelope theorem, continuously differentiable functions of $k, k_{0}, x, y$, and $\alpha$. The twice continuous differentiability of $V$ then follows from the fact that the composition of continuously differentiable functions is continuously differentiable. 


\section{Determinacy of Equilibria}

By considering savings functions $s$, we have reduced the problems of finding an equilibrium to the problem of finding an vector $\alpha$ that solves the $m$ equations $s\left(k_{0}, \theta, \phi, \alpha\right)=0$. In this section we use that fact to prove that for almost all endowments, there are a finite (and odd) number of equilibria.

From property (b) of Proposition 1, we see that $\sum_{i=1}^{m} s_{i}=0$. Consequently, it suffices to solve the system $s^{-m}=\left(s_{1}, s_{2}, \ldots, s_{m-1}\right)$, with one equation deleted. Moreover, by property (a), we may restrict a to lie on the unit simplex. Since $\sum_{i=1}^{m} \theta_{i}=1$, we may simply set $\theta_{m}=1-\sum_{i=1}^{m-1} \theta_{i}$, and let $\theta^{-m}=\left(\theta_{1}, \ldots, \theta_{m-1}\right)$. (Recall that $\theta_{i}$ is consumer $i$ 's share of the total stock of capital.) From property (d), we see that for fixed $k_{0}$ and $\phi$ we may solve $s^{-m}\left(k_{0}, \theta, \phi, \alpha\right)=0$ to find

$$
\theta^{-m}=f(\alpha)
$$

where $f$ is continuously differentiable. Indeed,

$$
\begin{aligned}
f_{i}(\alpha)=- & \left(\phi_{i} \bar{x} \partial V\left(\bar{k}_{0}, \bar{x}, 1, \alpha\right) / \partial x+y_{i} \partial V\left(\bar{k}_{0}, \bar{x}, 1, \alpha\right) / \partial y_{i}-\alpha_{i} \partial V\left(\bar{k}_{0}, \bar{x}, 1, \alpha\right) / \partial \alpha_{i}\right) / \\
& \left(\bar{k}_{0} \partial V\left(k_{0}, \bar{x}, 1, \alpha\right) / \partial k_{0}\right) .
\end{aligned}
$$

Notice that, for some values of $\alpha$, some components of $\theta^{-m}$ may be negative. Indeed, from property (c), we see that if $\alpha_{i}$ is zero, $\theta_{i}$ is always negative. Of course, no such a can arise in equilibrium.

For a two person economy $(m=2)$, we sketch $f$ in Figure 1 . In this case, the existence of an equilibrium for each $0 \leq \theta_{1} \leq 1$ follows from $f<0$ when $\alpha_{1}=0$ and $f>1$ when $\alpha_{1}=1$. In the general case, existence follows from Brouwer's fixed point theorem as, for example, in Varian (1984).

Let us call an equilibrium a regular if the $(\mathrm{m}-1) \times(\mathrm{m}-1)$ dimensional derivative matrix of $f$ with respect to $\alpha, D_{\alpha} f(\alpha)$, is nonsingular. We 
call a value $\theta^{-\mathrm{m}}$ regular if every corresponding equilibrium, $\alpha$, characterized by $\theta^{-\mathrm{m}}=\mathrm{f}(\alpha)$, is regular.

For fixed $\theta^{-m}$, can there be a sequence of equilibria $\alpha^{n} \neq a$ converging to a regular equilibrium $\alpha$ ? Clearly, $\left(\alpha^{n}-\alpha\right) /\left\|\left(\alpha^{n}-\alpha\right)\right\|$ has a convergent subsequence, converging to a vector $d$ with unit length. Moreover, $f\left(\alpha^{n}\right)=f(\alpha)=\theta^{-m}$ implies that the directional derivative $D_{\alpha} f(\alpha) d$ equals 0 . This contradicts the fact that $D_{\alpha} f(\alpha)$ is nonsingular. Consequently, every regular equilibrium has a neighborhood in which there are no other equilibria. This, together with the compactness of the simplex and the continuity of the equilibrium conditions, implies that, if $\theta^{-\mathrm{m}}$ is a regular value, there are only finitely many corresponding equilibria. Because of the boundary condition (c) it follows from in Proposition 1, index theory that the number of equilibria is odd (see, for example, Varian 1974). 
$-23-$

Figure 1: The Equilibrium Map 
In the two person case illustrated in Figure 1, the horizontal line $\theta_{1}=1 / 2$ illustrates a typical regular endowment. The slope of $f$ does not vanish where this line is crossed. Consequently, there are finitely many equilibria, and, since both the initial crossing of the line and the final crossing are from below by the boundary condition, it is clear that the number of equilibria is odd.

This figure illustrates why indeterminacy could arise for a measure zero set of initial values for $\theta_{1}$. Nothing rules out the possibility that $f$ is constant over some interval of $\alpha^{\prime} s$. If the economy happened to start with a value for $\theta_{1}$ equal to the value that $f$ takes on in this range, then there would be an infinite number of nearby solutions to the equations $f(\alpha)=\theta_{1}$. It is intuitively clear, however, that almost all of the possible choices of $\theta$ do not correspond to a flat in $\mathrm{f}$.

This intuition is made precise by Sard's theorem. It says that if a function $f$ that maps an open subset of $\mathrm{R}^{\mathrm{m}-1}$ (corresponding in this case to the interior of the simplex for a) to $\mathrm{R}^{\mathrm{m}-1}$ is continuously differentiable, then the set of regular values $\theta^{-\mathrm{m}}$ has full measure. In other words, for almost all $\theta^{-m}, D_{\alpha} f(\alpha)$ is nonsingular for every value of $\alpha$ with $f(\alpha)=\theta^{-m}$. In the example in the figure, $D_{\alpha} f(\alpha)$ is nonsingular if $f^{\prime}(\alpha) \neq 0$.

Since the set of regular endowments $\theta^{-m}$ has full measure for each fixed $k_{0}$ and $\phi$, it follows from Fubini's theorem that the set of all $\theta$, $\phi$, and $\mathrm{k}_{0}$ for which $\theta^{-\mathrm{m}}$ is a regular value also has full measure. Our arguments can be summarized in the following result:

Proposition 2: Assumptions 1 and 2 imply that, for almost all $\theta$, $\phi$, and $\mathrm{k}_{0}$, there is a finite (odd) number of equilibria. Furthermore, the equilibrium weights $\alpha$ vary continuously with $\theta, \phi$, and $k_{0}$ in some neighborhood of each equilibrium. 
Moreover, since $D_{\alpha} f(\alpha)$ is nonsingular at each such equilibrium, the implicit function theorem allows us to solve locally for $\alpha$ as a function of $\theta$, $\phi$, and $\mathrm{k}_{0}$ to do comparative statics.

\section{The Multisector Model}

To what extend does the determinacy of equilibrium depend on the special features of the one sector growth model? Although the notation and assumptions can be stated more simply in the one sector case, the reduction of finding equilibrium in a production economy with finitely many consumers to finding zeros of finitely many savings functions requires only that markets are complete and that the first and second welfare theorems hold. This, however, is true in a much more general settings. Moreover, the conclusion that equilibria are determinate for generic endowments is based only on the fact that the savings function is $c^{1}$. The important use of the one sector assumptions has been in proving the stronger proposition that the value function is $c^{2}$. Consequently, it is sufficient for the study of determinacy in a production economy to study the $c^{2}$ differentiability of the value function for that technology.

To what extent is the value function $c^{2}$ in a multisector model? Araujo and Scheinkman (1977) show that if the technology itself is sufficiently differentiable, a $c^{2}$ value function follows from a global turnpike theorem. In other words, if all socially optimal paths converge to a unique stationary state (which may, however, depend on the welfare weights), and suitable technical conditions are satisfied, equilibria are generically determinate. This, indeed, is the basis of the result in the one sector case. In a multisector model, a global turnpike theorem generally requires that the discount factor be close to one: see McKenzie $(1983,1986)$. 
On the other hand, if the discount factor is small, and suitable technical conditions are satisfied, Boldrin and Montrucchio (1988) have shown that the value function is $c^{2}$. This is true despite the fact that Boldrin and Montrucchio (1985) and Deneckere and Pelikan (1986) have shown that with the same small discount factors not only may optimal paths cycle, they may be chaotic. In other words, a $\mathrm{C}^{2}$ value function (and determinacy) do not require a global turnpike theorem.

This leaves one important gap: there is an intermediate range of discount factors for which it has not been shown that the value function is $c^{2}$. Moreover, there are no known counterexamples that do not violate conditions (such as strong concavity) that are part of the sufficient conditions for $\mathrm{C}^{2}$ differentiability of the value function with a finite horizon.

Given this gap, it is important to ask to what extent $c^{2}$ differentiability of the value function is actually needed. First, is $C^{1}$ differentiability of the savings function needed? Certainly, if the savings function is $c^{0}$, robust examples of indeterminacy exist: see Kehoe, Levine, Mas-Colell, and Zame (1986). If the savings function is Lipshitz continuous in the utility weights $\alpha$, however, Santos (1987) has shown that this suffices for determinacy. The argument is that of the previous section: Sard's theorem requires only Lipshitz continuity. On the other hand, Montrucchio (1986) has shown that the value function is $C^{1}$ Lipshitz in initial capital stocks under relatively general conditions. Although this is promising, the remaining gap is to show that the value function is also $C^{1}$ Lipshitz with respect to parameters such as $\alpha$. Again, neither a positive result nor a counterexample is available. 


\section{References}

Araujo, A., and Scheinkman, J. A. 1977. "Smoothness, Comparative Dynamics, and the Turnpike Property," Econometrica 45, 601-20.

Benveniste, L. M., and Scheinkman, J. A. 1979. "On the Differentiability of the Value Function in Dynamic Models of Economics," Econometrica 47, $727-32$

Bewley, T. 1980. "The Permanent Income Hypothesis and Long Run Economic Stability," Journal of Economic Theory 22, 252-92.

- 1982. "An Integration of Equilibrium Theory and Turnpike Theory," Journal of Mathematical Economics 28, 221-34.

Blume, L., Easley, D., and O'Hara, M. 1982. "The Characterization of Optimal Plans for Stochastic Dynamic Programs," Journal of Economic Theory 28, 221-34.

Boldrin, M., and Montrucchio, L. 1985. "The Emergence of Dynamic Complexities in Models of Optimization Over Time: The Role of Impatience," Rochester Center for Economic Research Working Paper no. 7. . 1986. "On the Indeterminacy of Capital Accumulation Paths," Journal of Economic Theory 40, 26-39.

. 1988. "On the Differentiability of Policy Functions," unpublished manuscript, University of California, Los Angeles.

Cass, D., 1965, "Optimal Growth in an Aggregate Model of Capital Accumulation," Review of Economic Studies 32, 233-42.

Deneckere, R., and Pelikan, S. 1986. "Competitive Chaos," Journal of Economic Theory 40, 13-25.

Harris, M. 1987. Dynamic Economic Analysis, Oxford University Press. Hildenbrand, W. 1974. Core and Equilibria of a Large Economy, Princeton. 
Kehoe, T. J., and Levine, D. K. 1985a. "Comparative Statics and Perfect Foresight in Infinite-Horizon Economies," Econometrica 53, 433-53.

- 1985b. "Empirical Implications of Complete Contingent Claims," unpublished manuscript, U.C.L.A.

. 1987. "The Economics of Indeterminacy in Overlapping Gener-

ations Models," unpublished manuscript, University of Minnesota.

Kehoe, T. J., Levine, D. K., Mas-Colell, A., and Zame, W. R. 1986. "Determinacy of Equilibrium in Large-Square Economies," Journal of Mathematical Economics forthcoming.

Kehoe, T. J., Levine, D. K., and Romer, P. M. 1987. "Steady States and Determinacy in Economies with Infinitely Lived Consumers," in Joan Robinson and Modern Economic Theory G. R. Feiwel (ed.), New York: MacMillan, forthcoming.

McKenzie, L. W. 1959. "On the Existence of General Equilibrium for a Competitive Market," Econometrica 27, 54-71. . 1983. "Turnpike Theory, Discounted Utility, and the von Neumann Facet," Journal of Economic Theory 30, 330-52. . 1986. "Optimal Economic Growth and Turnpike Theorems," in Handbook of Mathematical Economics 3, K. J. Arrow and M. D. Intriligator (eds.), Amsterdam: North-Holland.

Montrucchio, L. 1986. "Lipshitz Continuous Policy Functions for Strongly Concave Optimization Problems," unpublished manuscript, Politecnico di Torino.

Muller, W. J., and Woodford, M. 1986. "Stationary Overlapping Generations Economies with Production and Infinite-Lived Consumers," Journal of Economic Theory, forthcoming. 
Negishi, T. 1960. "Welfare Economics and Existence of Equilibrium for a Competitive Economy," Metroeconomica 23, 92-7.

Romer, P. M., and Shinotsuka, T. 1988. "The Kuhn-Tucker Theorem Implies the Transversality Condition at Infinity," unpublished manuscript, University of Rochester.

Santos, M. 1987. communication to the authors.

Varian, H. R. 1974. "A Third Remark on the Number of Equilibria of an Economy," Econometrica 43, 985-86.

Varian, H. R. 1984. Microeconomic Analysis, W. W. Norton.

Weitzman, M. 1973. "Duality Theory for Infinite Horizon Convex Models." Management Science $19,783-89$. 
